\title{
Race/classe/genre. Parcours dans l'historiographie américaine des femmes du Sud autour de la guerre de Sécession
}

\section{Céline Bessière}

\section{(2) OpenEdition \\ Journals}

Édition électronique

URL : https://journals.openedition.org/clio/591

DOI : 10.4000/clio.591

ISSN : 1777-5299

Éditeur

Belin

Édition imprimée

Date de publication : 1 avril 2003

Pagination : 231-258

ISBN : 2-85816-663-3

ISSN : 1252-7017

Référence électronique

Céline Bessière, «Race/classe/genre. Parcours dans I'historiographie américaine des femmes du Sud autour de la guerre de Sécession », Clio. Histoire, femmes et sociétés [En ligne], 17 | 2003, mis en ligne le 27 novembre 2006, consulté le 24 avril 2022. URL : http://journals.openedition.org/clio/591 ; DOI : https://doi.org/10.4000/clio.591

Ce document a été généré automatiquement le 24 avril 2022.

Tous droits réservés 


\title{
Race/classe/genre. Parcours dans l'historiographie américaine des femmes du Sud autour de la guerre de Sécession
}

\author{
Céline Bessière
}

1 La première tâche des sociologues qui se sont intéressés à la variable genre ${ }^{1}$ a consisté à dénaturaliser leur objet et à montrer, avant tout, que les inégalités entre les sexes étaient bien des inégalités sociales. Ce travail de dénaturalisation a été réalisé cependant en concurrence, contre, les autres variables sociales. Les sociologues du féminin / masculin ont entrepris, en priorité, de réparer « l'oubli des rapports sociaux entre les sexes $»^{2}$, au détriment de l'étude de l'articulation entre différents types d'inégalités.

2 Les ressources tant empiriques que théoriques pour aborder le problème de l'articulation - qui suppose la description de rouages concrets entre différents types d'inégalités sociales - sont bien minces. Certes, les féministes marxistes ont cherché dans les années 70 quel était le premier type de domination - le patriarcat ou le capitalisme ${ }^{3}$. Les discussions interminables, très théoriques, engendrées par ce type d'interrogation sont hélas peu fécondes en termes de travaux empiriques en sciences sociales ${ }^{4}$. De même, la tentative plus récente de Pierre Bourdieu, en 1998, d'intégrer la question des inégalités de sexe à une théorie de la domination s'avère peu opératoire du point de vue de l'articulation de différents types d'inégalités. La domination masculine est présentée comme une forme de domination sociale parmi d'autres dont les mécanismes sont transposés dans un nouveau domaine. Cette relation d'homologie entre différents types de domination évacue du texte de Bourdieu toute question de l'articulation des inégalités, qui toutes homogènes peuvent être simplement cumulées comme une somme d'atouts ou de handicaps ${ }^{5}$.

3 Penser ensemble domination de classe et domination de genre revient ainsi, le plus souvent, à subsumer l'un des types de domination à l'autre. Un bon exemple de ce type 
de raisonnement, longtemps dominant dans la sociologie française, est l'article de JeanClaude Passeron et François de Singly, paru en 1984 dans la Revue française de sciences politiques: "Différences dans la différence ». Cet article se propose d'analyser les " effets croisés » du sexe et de la classe sociale sur un ensemble de pratiques et de représentations juvéniles. D'emblée le lecteur est prévenu: "les trois principes de variation de la socialisation, que l'on analyse dans cet article - classe sociale, sexe, domaine d'action ou de représentation - ne sont, ni logiquement, ni sociologiquement, de même nature. On n'attendra donc pas leur croisement dans n'importe quel ordre. La classe sociale commande, ici, l'ordre de l'analyse parce qu'elle constitue un cadre de socialisation particulièrement différenciateur [...], tandis que le sexe ou l'âge occupent une toute autre position puisqu'ils spécifient la socialisation dans le cadre de chaque milieu social, selon les individus à socialiser ». Certaines catégories - les classes sociales - apparaissent d'emblée et de manière non justifiée comme plus "sociologiques » que d'autres, alors que le sexe et l'âge sont reléguées au rang de variables individuelles. La relecture de ce texte nous fait percevoir a posteriori combien tout le travail de sociologisation de la variable sexe est véritablement un préalable nécessaire aux questions d'articulations. Si le genre est du social, nous ne dressons pas ici une équivalence entre le "social" et les inégalités de classe ou la stratification socioéconomique. Il s'agit bien d'articuler différents types de domination sociale sans effacer un des membres. Même si le terme d'articulation gagnerait à être précisé, notre intuition de départ est que face à différentes sortes de dominations, il faudrait préciser les mécanismes concrets, les chevilles entre les différents éléments à articuler. Comment sortir des logiques d'encastrement qui hiérarchisent les formes de domination, minorant, oubliant ou neutralisant l'une d'entre elles?

4 Face à ces questions, il est légitime de se tourner vers les sciences sociales aux EtatsUnis où les problématiques en termes d'articulation des variables Race/Classe/Genre sont d'usage fréquent et prennent des formes très diverses. Il s'agit parfois d'un questionnement "haché", découpé en départements d'université (women's studies, african american studies ${ }^{6}$, etc.), qui invite tout chercheur à s'interroger systématiquement : mais qu'en est-il des femmes ? et des noirs ? et des pauvres ? etc. Ce type de questionnement (souvent caricaturé et critiqué en France) issu de la professionnalisation des questions de genre, de classe, de race présente la grande faiblesse de superposer des catégories d'analyse "toutes faites" à la réalité sociale sans s'intéresser à la construction même de ces catégories, ni même à leur pertinence. Surtout, il tend à isoler les questions ayant trait aux hommes et aux femmes, aux blancs et aux noirs, aux riches et aux pauvres. Utiliser le concept de genre pour une interrogation du type « et qu'en est-il des femmes? » clôt le monde des femmes sur luimême et ne fait qu'ajouter un nouveau compartiment à la discipline historique ${ }^{7}$. Il va de soi que nous ne considérons pas ce simple "et" comme une analyse suffisante de l'articulation entre classe, race, et genre.

5 Loin des approches catégorielles statiques, nous pensons, au contraire, que les catégories de race, de genre et de classe, utilisées comme un outil de réflexion historique et sociologique permettent d'éclairer et de penser des aspects de la société américaine dans son ensemble (ou de la société française, sous réserve d'adaptations des catégories aux situations sociales à étudier). Nous pouvons ici nous appuyer sur les travaux du linguistic turn qui reprennent à leur compte des concepts de la philosophie post-structuraliste ${ }^{8}$. Le déconstructionnisme invite à percevoir en quoi les concepts de genre, de classe et de race ne sont pas des entités fixes, naturelles mais avant tout des 
constructions sociales et historiques, arbitraires et instables, des outils de polarisation de la domination et du pouvoir dans la sociétés. Les analyses du pouvoir de Michel Foucault permettent de considérer non pas un pouvoir unifié, cohérent, centralisé, et une opposition binaire et globale entre dominants et dominés, mais au contraire d'étudier au niveau local des constellations de pouvoir dispersées, des configurations de relations inégalitaires constituées dans des champs de forces sociaux. Nous évitons ainsi le piège de "l'encastrement" d'une forme de domination dans l'autre, puisqu'on peut envisager une pluralité de régimes de pouvoir non nécessairement convergents ${ }^{10}$.

Nous sommes invités à penser l'articulation entre des inégalités de genre, de race, et de classe en termes de configurations historiques concrètes de relations de pouvoir. D'où l'idée de travailler plus précisément sur une période historique et une région: le Sud des États-Unis autour de la guerre de Sécession ${ }^{11}$. L'histoire des femmes du Sud apparait comme un cas limite, permettant d'articuler idéalement (au sens weberien d'idéal type) différents types de régimes de pouvoir. Ce champ historiographique s'est en effet constitué et développé en suivant les lignes de l'articulation entre race, genre et classe (l'ordre chronologique étant ici important).

Une historiographie qui épouse les contours de l'articulation race, genre et classe

La southern women's history apparaît comme un champ récent et d'ampleur limitée par comparaison avec d'autres grands secteurs de l'histoire des femmes aux États-Unis. On peut en situer la naissance au début des années $1970^{12}$ avec l'ouvrage précurseur d'Anne Firor Scott, The Southern Lady, mais il faut attendre le milieu des années 1980 pour qu'un véritable domaine de recherche sur l'histoire des femmes du Sud s'ouvre et $s^{\prime}$ institutionnalise ${ }^{13}$. Avant d'entrer dans le détail des textes, il importe de remarquer combien ce champ historiographique s'est constitué en opposition avec l'histoire de l'esclavage d'une part, et l'histoire des femmes d'autre part: deux histoires qui tendaient à escamoter la question des femmes du Sud.

L'occultation des femmes par la nouvelle histoire de l'esclavage (1960-1970)

Jusque dans les années 1960, la thèse de Stanley Elkins domine l'histoire du Vieux Sud: elle met l'accent sur l'extrême brutalité de l'esclavage et sur son caractère totalitaire. Les esclaves sont perçus comme de purs objets de l'oppression des planteurs, des victimes dépersonnalisées, dépendantes et infantilisées: des $S a m b o^{14}$. Par ailleurs, il faut faire le lien entre la théorie d'Elkins et la thèse, là encore dominante (en particulier après la publication du rapport Moynihan en $1965^{15}$ ), de la femme noire matriarche : l'esclavage détruit la structure de la famille noire, il ôte tout pouvoir aux hommes esclaves qui jusque dans leurs familles sont dominés par les femmes.

En réponse à la thèse d'Elkins et de Moynihan, de jeunes historiens, se réclamant de la «nouvelle histoire sociale », mettent l'accent dans les années 1960-1970, sur les marges de liberté, les marges de manœuvre (agencies) et la cohérence des "communautés " d'esclaves qui sont décrites sous le vocable de la "solidarité ", la "sociabilité », les "liens de parenté », la " culture », «l'autonomie » face aux planteurs. Ce sont les New Slavery Studies ${ }^{16}$ et l'apogée de l'histoire du Vieux Sud. Prendre "l'histoire d'en bas " lorsqu'il s'agit du Sud revient à déplacer l'analyse, du monde du planteur vers le monde des esclaves. Pour ce faire, ces chercheurs exploitent des sources inédites à côté des livres de plantation (qui retracent la comptabilité et la gestion de la plantation, du point de vue du planteur): des contes, chansons, autobiographies et entretiens avec d'anciens esclaves réalisés dans les années 1930, dans le cadre du Federal Writers Project $(F W P)^{17}$. 
10 La nouvelle histoire de l'esclavage a constitué un point de départ pour l'histoire des femmes du Sud à la fois blanches et noires. Son apport est considérable du point de vue du déplacement des problématiques - le décentrement du monde du planteur vers celui des esclaves - mais aussi du point de vue méthodologique par l'usage de sources nouvelles et le traitement original des archives. Les historiennes des femmes du Sud sont, cependant, d'emblée critiques par rapport à cette histoire sociale de l'esclavage (faite principalement par des hommes), qui dans sa volonté de mettre à jour des communautés solidaires d'esclaves, présente des familles nucléaires, calquées sur les familles blanches, où domine le père. La nouvelle histoire de l'esclavage, pour mieux lutter contre la figure de Sambo, a utilisé la métaphore de la «masculinité » (manhood), quitte à ignorer ou minimiser le rôles des femmes esclaves ${ }^{18}$.

L'oubli des femmes du Sud par l'histoire des femmes (1970-1980)

11 Les premiers travaux retentissants d'histoire des femmes mettent l'accent sur une culture féminine s'épanouissant au XIXe siècle, au travers une séparation entre des mondes masculin et féminin ${ }^{19}$ : les concepts de true woman, separate spheres et woman's culture sont alors dominants. Alors même que le cadre spatio-temporel est bien délimité - il s'agit de l'étude de classes moyennes victoriennes du Nord des Etats-Unis - ces travaux deviennent de véritables références pour l'histoire des femmes qui se développe au début des années 80. Critiqués pour leur essentialisme, on peut remarquer aussi que ces travaux rendent peu compte de la situation des femmes du Sud. La séparation victorienne des espaces masculin et féminin, et le développement du féminisme qui en résulte ne permettent pas de comprendre la position de femmes blanches et noires vivant sous un même toit et sous l'autorité d'un planteur (père, mari, maitre). Catherine Clinton, l'une des pionnières de l'histoire des femmes du Sud, dénonce ainsi ce qu'elle nomme en 1982, New Englandization of American women's history: alors que les femmes du Nord ont créé, au XIXe siècle, une culture propre qui sert de fondement au féminisme, les femmes du Sud isolées dans leur plantation sont incapables de former de telles identités collectives. La southern women's history a participé à la critique du concept de sisterhood et à l'élaboration d'une histoire des femmes plus centrée sur les différences parmi les femmes et les différences entre hommes et femmes ${ }^{20}$.

Naissance (1970-1980) puis essor (1990-2000) de l'histoire des femmes du Sud

12 L'histoire des femmes du Sud s'est donc formée en appui mais aussi contre la nouvelle histoire de l'esclavage et contre l'histoire des femmes du Nord. Un premier groupe de femmes blanches universitaires se concentre principalement sur l'étude des femmes de planteurs de la période antebellum en utilisant des sources qui n'avaient jamais été dépouillées jusqu'alors : correspondances et journaux intimes de femmes, mémoires de femmes après la guerre civile, articles de journaux de l'époque ${ }^{21}$. Cette histoire des femmes de planteurs s'écrit en correspondance avec les ouvrages de la nouvelle histoire sociale de l'esclavage, ce qui constitue un premier moment de l'articulation entre race et genre. Parallèlement, au milieu des années 1980, des historiennes noires ${ }^{22}$ vont s'intéresser aux femmes esclaves jusqu'ici peu étudiées - et par la southern women's history qui se focalise sur les femmes de planteurs, et par la nouvelle histoire sociale de l'esclavage dont les objets fétiches sont plutôt les communautés ou les familles d'esclaves.

13 Un des objectifs commun à ces deux ensembles de travaux est avant tout de rompre avec les nombreux mythes d'un Vieux Sud nostalgique - The Happy Plantation - qui ont 
proliféré, notamment dans la littérature et au cinéma, et sont largement passés dans le sens commun ${ }^{23}$. Il s'agit de décrire des pratiques, afin de déconstruire les mythes, et tout particulièrement les mythes attachés à des personnages sexuellement définis. Anne Firor Scott, dès The Southern Lady, en 1970, expose la dure vie des femmes de planteurs dans un système patriarcal : elles sont peu éduquées, vivent dans le climat pestilentiel de la côte, subissent des accouchements à répétition, font face à un double standard en matière de sexualité etc. Elle remet en cause à la fois le mythe de la femme de planteur, incarnant la maternité glorifiée, the Queen of the Home, mais aussi le mythe du Gentleman qui est censé protéger sa «famille blanche et noire ». On peut remarquer cependant, à travers le titre de son ouvrage, The Southern Lady ${ }^{24}$, que Scott demeure prisonnière de l'imagerie de «la» femme du Sud, sans préciser qu'elle ne s'intéresse qu'à une frange bien spécifique de la population des femmes : les très riches femmes de planteurs, la catégorie sociale de Scarlett O'Hara. L'histoire des femmes du Sud en poursuivant ce travail de description a eu l'intérêt de montrer qu'il n'existe pas un seul modèle de femme (ou d'homme) qui par son essence même serait l'incarnation du Vieux Sud, mais une pluralité de trajectoires sociales possibles.

Si la nouvelle histoire de l'esclavage est revenue sur le mythe de Sambo (l'esclave docile), ce sont des historiennes noires qui se sont attaquées à celui du matriarcat des femmes esclaves. L'article d'Angela Davis, «Reflections on the Black Woman's Role in the Community of Slaves » en 1971, joue ici un rôle primordial: elle y dénonce les enjeux politiques d'un tel mythe, destiné à diminuer les capacités de résistance des femmes noires (dans la période de lutte pour les droits civils), en leur opposant l'idéal de la suprématie masculine. Sur le flanc historique, elle avance également l'impossibilité du matriarcat des femmes noires au sein du système esclavagiste qui ne peut reconnaître de tels symboles d'autorité, des formes de pouvoir du côté des esclaves, fussent-elles des femmes ${ }^{25}$.

Aujourd'hui, les travaux les plus novateurs quant aux sources utilisées, prennent en compte des personnages oubliés par les grands mythes du Vieux Sud. Il s'agit de mettre l'accent sur la diversité socio-économique et culturelle à la fois parmi les Blancs et parmi les Noirs. Des recherches récentes décrivent la position des Blancs pauvres - nonslaveholding Whites, yeomen - à travers l'étude d'archives judiciaires principalement ${ }^{26}$. La catégorie "blanc" s'avère dans cette perspective beaucoup plus diversifiée socioéconomiquement que dans les imageries du Vieux Sud. Des travaux vont même jusqu'à distinguer ethniquement et religieusement les populations d'origine allemande, italienne, irlandaise, française, créole, juive, quaker ou catholique ${ }^{27}$. Même à l'intérieur du monde des esclaves, on peut faire émerger des lignes de partage socio-économiques. La plus répandue et connue est celle qui sépare la main-d'œuvre agricole (field hands) des esclaves qui travaillent dans la Big House, mais on peut aussi distinguer la simple main d'œuvre agricole, des esclaves qui ont une compétence technique, artisanale particulière, ou prendre en compte les différentes origines ethniques des esclaves ${ }^{28}$. Des recherches se sont également intéressées à des familles de Métis $^{29}$ ou à des cas isolés en marge du système racial et social ${ }^{30}$.

Au-delà du constat de la formation et de l'institutionnalisation d'un champ de recherche avec ses points de repère, ses clivages, ses références, on mesure à présent combien l'histoire des femmes du Sud est cumulative en ce qui concerne la question de l'articulation entre race, genre et classe. 
Comment faire une histoire de l'imbrication des rapports de genre, de race et de classe dans le Vieux Sud?

Deux grandes questions se sont simultanément posées à la southern women's history. Des historiennes blanches ont débattu sur le degré d'adhésion et / ou de résistance des femmes de planteurs au système esclavagiste et patriarcal. Des historiennes noires se sont demandées quelles étaient les positions spécifiques des femmes esclaves à la fois par rapport aux hommes esclaves et par rapport à la classe des planteurs (hommes et femmes), quelles marges de manœuvre et quels moyens de résistance s'offraient à elles. Nous faisons le pari qu'à travers les débats qui animent les réponses à ces deux grandes questions, apparaissent des analyses fécondes quant à la possibilité d'articuler des rapports de genre, de race, et de classe dans une même histoire «inclusive ». En effet, ces questions reviennent à se demander d'une part, quelle est la position spécifique de femmes blanches qui bénéficient de privilèges de classe tout en vivant dans un système patriarcal, d'autre part, quelle est la position de femmes noires qui sont dominées dans la configuration race, classe et genre des plantations.

Femmes de planteurs, patriarcat et esclavage

18 Les questions de l'adhésion et/ou de la résistance des femmes de planteurs à l'institution esclavagiste et au modèle patriarcal se sont posées dès l'ouvrage fondateur d'A. F. Scott en 1970. Scott conduit une thèse nuancée sur le rapport des femmes de planteurs à l'esclavage. Si un certain nombre de femmes de l'élite blanche ont des doutes moraux sur l'institution esclavagiste et entretiennent des attachements personnels profonds avec certain(e)s esclaves, elles bénéficient cependant matériellement de l'esclavage en tant que membres de la classe des planteurs.

Sur la question de la résistance au patriarcat, Scott est en revanche "optimiste". Certes, les femmes de planteurs ne peuvent pas lutter directement contre le système patriarcal, mais elles profitent de situations de veuvage pour s'émanciper, ou participent à des associations chrétiennes où elles bénéficient d'une certaine autonomie et entendent les premiers échos des débats sur les droits des femmes, qui naissent dans le Nord au XIXe siècle. Sur ce point, l'analyse de Scott sera invalidée par les recherches fouillées, en 1985, de Jean Friedman sur le lien entre les femmes et les églises dans le Sud. Les églises évangéliques dans le cadre d'un Sud rural où les familles sont isolées apparaissent comme des centres de sociabilité qui favorisent les liens familiaux, bien plus que les liens d'amitié entre femmes. L'analyse fine de procès religieux montre combien un contrôle strict des mœurs au sein de chaque église est maintenu par les hommes, avec une sévérité toute particulière envers les comportements déviants des femmes. Loin de constituer des lieux de sociabilité et d'émancipation féminine comme le pensait Scott, les églises empêchent la constitution d'associations féminines indépendantes au même rythme que dans le Nord.

Les deux questions de l'adhésion et de la résistance à l'esclavage et au patriarcat ont été réexaminées par Catherine Clinton, dans sa thèse très controversée, en $1982^{31}$. La démonstration menée par Clinton s'appuie sur une analogie entre l'oppression des esclaves et l'oppression des femmes blanches dans les plantations : « Le coton est roi, le planteur fait la loi : les femmes blanches et les esclaves servent tous le même maitre " (p. 35). En s'opposant à Scott, Clinton soutient que les femmes de planteurs se pensaient avant tout comme des opprimées, des victimes du système patriarcal de la plantation et non comme bénéficiant du système, en tant que maîtresses. Elles n'ont aucun moyen de résistance contre leurs oppresseurs (les hommes), et se tournent au contraire contre 
les femmes esclaves qu'elles considèrent comme la source de leurs maux. Un des fondements de leur opposition à l'esclavage serait ainsi leur jalousie sexuelle envers les femmes esclaves : c'est la construction de la figure de Jezebel (le stéréotype de la femme esclave lubrique et séductrice ${ }^{32}$ ). Bref, elles se trompent d'ennemi en ne parvenant pas à lier leur sort avec celui des femmes noires.

21 On peut remarquer ici, que les analyses de Scott comme celles de Clinton sont fortement imprégnées par l'histoire des femmes du Nord. Ainsi, Scott écrit des analyses parallèles à celles de Nancy Cott sur les solidarités entre femmes dans des institutions féminisées (les églises) et la thèse de Clinton sur l'analogie entre esclaves et femmes de planteurs fait directement écho à la thématique de la solidarité entre femmes (sisterhood) ${ }^{33}$. Pourtant, les rapports sociaux qui prennent corps dans le Vieux Sud ne se plient guère à cette problématique qui présuppose avant tout des points communs entre toutes les femmes ${ }^{34}$.

Le livre d'Elizabeth Fox-Genovese, Within the Plantation Household: Black and White Women in the Old South, qui paraît en 1988, constitue une charnière pour l'histoire des femmes du Sud à cet égard. Le concept central qui organise l'ouvrage est celui de maisonnée (household ${ }^{35}$ ), qui permet d'appréhender dans une même entité le planteur et sa famille, ainsi que les esclaves : «l'unité sociale de base, dans laquelle les membres, volontairement ou sous contrainte, mettent en commun leurs revenus et leurs ressources» (p. 31). À ce concept de maisonnée, Fox-Genovese associe celui de gender comme catégorie sociale et historique d'analyse : elle étudie ainsi à la fois les relations entre hommes et femmes, du point de vue des pratiques (division du travail, division des rôles sexués) et des représentations (stéréotypes de genre). Ce double choix conceptuel renouvelle le champ de l'histoire des femmes du Sud: il ne s'agit plus d'analyser les femmes de planteurs seules, mais les «maitresses» en relation à la fois avec leurs mari, père, frères (les planteurs) et les esclaves hommes et femmes dans l'entité maisonnée ${ }^{36}$.

L'identité de Lady (gracieuse, délicate, dévouée à sa famille, cultivée, élégante et protégée par les hommes de son entourage), terme central de la définition des femmes de l'élite blanche, est une identité de genre construite racialement et socialement. Par exemple, il est totalement implicite qu'une femme pour être véritablement une Lady, doit avoir des esclaves à sa disposition: cette évidence ressort dans les écrits des femmes de planteurs elles-mêmes, mais aussi dans les textes officiels émanant des idéologues pro-esclavagistes, qui présentent l'esclavage comme une manière d'élever la condition des Ladies, déchargées des tâches les plus ingrates par leurs esclaves. L'identité de Lady, une convention de genre, exprime en même temps que la féminité, la blancheur et une certaine position sociale dans le Vieux Sud esclavagiste.

De cette lecture du genre en relation avec les catégories de race et de classe découle la thèse de Fox-Genovese en matière de résistance des femmes de planteurs au système patriarcal esclavagiste. On se souvient que Scott percevait des signes d'opposition. Clinton au contraire soutenait qu'aucune résistance n'était possible pour les femmes blanches parce qu'elles étaient totalement opprimées par le système. Fox-Genovese insiste également sur l'impossibilité de la résistance des Ladies au système, mais pour des raisons différentes de Clinton. Les femmes de planteurs ne remettent jamais en cause politiquement le système social du Vieux Sud qui dans son aspect patriarcal, certes, les domine, mais leur assure leur position sociale privilégiée et se trouve au fondement de leur définition de genre, en tant que Lady. 

de pouvoir non-convergents fondés sur la classe, la race (les femmes de l'élite blanche sont selon ces deux coordonnées dans une position dominante, ce qui leur rend difficile toute opposition au système esclavagiste dont elles bénéficient) et le genre (les femmes de planteurs, en tant que femmes, sont ici en position de subordination). On ne peut ni travailler sur les femmes de l'élite comme une catégorie séparée du reste de la société esclavagiste, ni travailler à partir du concept de solidarités entre femmes (sisterhood) : les femmes blanches et noires sont simultanément réunies dans la plantation et écartées dans des mondes bien séparés et antagonistes ${ }^{37}$. Seule une analyse en termes de configurations qui accepte la complexité, les tensions et les contradictions entre différents types de régimes de pouvoir peut rendre compte de ce type de situations sociales.

Comment pousser les femmes esclaves dans les plantations?

Une première manière de décrire la position des femmes esclaves est de souligner la double oppression qui pèse sur elles, en tant qu'esclaves et en tant que femmes. C'est la thèse du cumul des handicaps (double burden) ${ }^{38}$. En raison de l'esclavage, les femmes noires sont tenues de travailler pour leur maître autant que les hommes. Jacqueline Jones note que la plupart du temps les femmes étaient comptées comme $3 / 4$ hand, mais que plus la plantation était petite, plus le travail était dur et varié pour les femmes, incluant les tâches les plus physiques (défricher des terres ou couper, transporter du bois). Même si les planteurs ont comme dilemme d'exploiter au maximum le travail des femmes ou leur accorder du repos pendant leur période de grossesse, être enceinte constitue toujours pour les esclaves une forme de travail, puisque le statut d'esclave se transmettant par les femmes, elles reproduisent ainsi la main d'œuvre de la plantation. Mais les femmes esclaves doivent aussi assumer des tâches domestiques, typiquement féminines, dans le quartier des esclaves : préparer la nourriture, s'occuper des enfants, entretenir les cabines, etc. D'où l'accent sur le double burden : rapports de race-classe et de genre s'additionnent pour décrire la position des femmes esclaves à la fois par rapport aux hommes esclaves et par rapport aux femmes de planteurs.

Deborah Gray White, en 1985, décrit la position des femmes esclaves en de tout autres termes. En travaillant dans les champs aussi durement que les hommes, les femmes esclaves sont «libérées» de leur statut de femme qui les confinerait (comme leur maitresse) dans l'espace domestique. Dans une argumentation qui rappelle celle des New Slavery Studies, mais centrée sur les femmes, White met l'accent sur des sphères d'autonomie, des réseaux de sociabilité entre femmes esclaves, qui travaillent le plus souvent ensemble dans des groupes non-mixtes. Elle décrit des relations privilégiées avec les enfants et une certaine indépendance par rapport aux hommes, ce qui lui permet de parler de famille matrifocale. Dans cette perspective, l'esclavage, en tant que rapport de domination qui pèse à la fois sur les femmes et les hommes noirs, prive les hommes des moyens de la domination masculine: ils ne sont pas propriétaires, ils n'assurent pas la survie économique de leur foyer, le divorce est relativement facile, etc. Dans cette situation, l'esclavage réduit la domination par le genre, parmi les esclaves et White qualifie les relations entre hommes et femmes esclaves "d'égales ».

Cette argumentation a été l'objet de nombreux débats. Jones s'oppose à l'usage du terme égalité pour décrire les relations entre hommes et femmes esclaves. Certes, les hommes esclaves ne peuvent pas asseoir une domination sur leurs femmes qui reposerait sur une supériorité économique, puisqu'ils ne possèdent rien, mais on ne 
peut pas pour autant parler d'égalité, surtout si on prend en compte la vulnérabilité des femmes esclaves aux coups et aux viols de la part des planteurs. Jones remarque aussi combien à l'intérieur des quartiers d'esclaves une stricte division sexuelle du travail était de mise. Dans les interviews FWP, auprès d'anciens esclaves, elle met ainsi en avant deux traits récurrents: un profond respect, envers le travail des femmes esclaves, mais aussi un réel « mépris » pour les hommes qui réalisent (souvent de force) un travail étiqueté comme féminin: cuisine, couture, nettoyage, entretien du linge et des enfants, etc.

On peut ajouter, à l'inverse, que la domination de genre permet aux femmes d'échapper, dans une certaine mesure, à l'oppression de l'esclavage. On a vu avec Jones que les femmes assuraient le poids des corvées domestiques dans les quartiers d'esclaves. Cependant, le travail domestique, dans la situation particulière de l'esclavage acquiert un statut bien particulier. En comparaison avec le travail sur la plantation, les tâches ménagères ont pour finalité la communauté des esclaves et non le planteur. Dans la perspective marxiste d'Angela Davis, c'est le seul travail qui ne soit pas aliéné par la classe des planteurs. Plus récemment, Stevenson, en 1996, a montré à partir de l'étude d'entretiens FWP combien les femmes esclaves mettaient en œuvre des pratiques de résistance contre l'esclavage qui s'appuyaient sur leur position de femme. Non seulement elles s'autorisent, mais aussi valorisent comme partie prenante de leur féminité, des comportements de résistance face aux planteurs, des mensonges, des feintes pour échapper au travail, des vols de nourriture. On peut dire ici que le genre «atténue » dans une certaine mesure la pression de l'esclavage. Mais les nombreux cas d'abus sexuels perpétrés par des hommes blancs sur les femmes esclaves nous ramènent à la thèse du double burden.

Ces débats sont d'un apport considérable pour l'appréhension dans une même analyse de rapports de pouvoir fondés sur les classes, les races et les genres. Esclavage et domination de genre semblent à première vue se renforcer mutuellement, mais on peut aussi dans une certaine mesure montrer que l'esclavage tempère la domination des hommes sur les femmes esclaves, ou que le genre atténue en partie la pression de l'esclavage sur les femmes noires. Cette controverse, au milieu des années 80 , nous montre aujourd'hui les limites d'une conception de l'articulation race/classe/genre fondée sur la sommation des atouts et des handicaps dont disposent les femmes esclaves (et plus généralement toute population prise dans des régimes contradictoires de pouvoir). Il faut revenir, en effet, sur les outils conceptuels dont disposent les historiennes de l'esclavage au milieu des années 1980. D. White, une des participantes actives à ces débats, dans la nouvelle édition de Ar'n't I a woman en 1999, revisite son ouvrage de 1985 en mettant à jour les présupposés qui sous-tendaient alors les recherches sur les femmes esclaves. Elle reconnaît que si en 1985, il était relativement facile, à partir des sources, de faire une lecture en termes de différences (entre hommes et femmes esclaves et entre femmes noires et blanches), conceptualiser ces différences s'avérait beaucoup plus difficile ${ }^{39}$. Elle reprend ainsi des passages du texte de 1985 qu'elle ne réécrirait plus de la même manière aujourd'hui. Par exemple : « La condition de la femme esclave est seulement un cas extrême de ce que les femmes en tant que groupe ont subi aux Etats-Unis, car le racisme, bien que tout aussi pénétrant que le sexisme, était aussi plus virulent » (p. 15). Dans ce type de proposition, le racisme et le sexisme peuvent être ajoutés pour produire un préjudice encore plus grand que le racisme ou le sexisme seuls. 
31 L'articulation entre race, classe et genre en 1985 était pensée en termes de cumul des handicaps ou d'atténuation des formes de domination entre elles. On retrouve le même présupposé dans les textes de J. Jones : « La position économique des femmes noires par rapport aux femmes blanches est analogue à la position relative des femmes par rapport aux hommes, parce que les femmes noires ont été limitées au travail des Noires, qui est moins bien rémunéré que le travail des Blanches » (p. 4). Ce qui soustend ces opérations d'addition et de soustraction est une homologie entre les différentes formes de domination, ce qui permet de dresser des équivalences entre oppression issue de l'esclavage, et domination de genre.

Ce type de raisonnement qui dissocie dans un premier temps les rapports de genre des rapports de classe et de race et qui ensuite articule les différentes formes de domination sous le mode de l'addition ou de la soustraction repose en fait sur une fiction; la fiction qu'il existerait des femmes et des hommes abstraits définis par un sexe, indépendamment des rapports de race et de classe (comme dans un raisonnement statistique «toutes choses égales par ailleurs»). De nombreuses femmes, comme de nombreux hommes, n'ont jamais bénéficié de tous les éléments de cette fiction ${ }^{40}$. Les femmes noires ne vivent tout simplement pas le sexisme de la même manière que les femmes blanches. De la même façon, les femmes noires ne vivent pas le racisme de la même façon que les hommes noirs. «Le viol des femmes esclaves, leur dur travail, la négation de leur beauté, le désintérêt porté à leur grossesse, la vente de leurs enfants constituent simultanément des manifestations du racisme et du sexisme qui pèsent sur elles, et non pas une forme extrême de l'un ou de l'autre $»^{41}$.

On ne peut donc pas dissocier la catégorie de genre de la catégorie de race et de classe. Seule l'articulation de ces rapports de pouvoir permet de comprendre la position toute spécifique des femmes esclaves: « Nous comprenons maintenant que la race, la classe, le genre, la sexualité et toute autre catégorie identitaire n'existent pas indépendamment l'une de l'autre. Elles ne sont pas non plus en concurrence pour une éventuelle suprématie, mais elle se renforcent, se chevauchent, s'entrelacent l'une à l'autre $»^{42}$. En reprenant l'expression de Danièle Kergoat, on peut parler de construction consubstantielle de la classe, de la race et du sexe dans des configurations spécifiques, ce qu'elle appelle des systèmes intégrés ${ }^{43}$.

Comment évoluent les configurations race/classe/genre pendant et après la guerre de Sécession ${ }^{44}$ ?

34 Étant donné l'imbrication des rapports de race, de classe et de genre, on peut faire l'hypothèse que la guerre de Sécession, qui constitue une remise en cause profonde du système social du Sud - par la présence matérielle de la guerre, mais surtout par l'émancipation des esclaves en 1865 - va altérer les configurations décrites ci-dessus. Nous commençons ici à appréhender l'articulation entre les inégalités de genre et les autres inégalités sociales dans la durée, de façon dynamique, et en prenant le temps comme principe de comparaison.

Identités de genre pendant la guerre de Sécession : l'exemple des Ladies

Quelques ouvrages ont traité dans les années 1960 et 1970 de la participation des femmes blanches de l'élite à l'effort de guerre, en soulignant l'importance de leur soutien à la cause confédérée ${ }^{45}$. Ces travaux ont mis l'accent sur l'expérience de la guerre comme tournant décisif pour l'émancipation des femmes de planteurs. Dès 1862, les lois sur la conscription enrôlent plus de trois quarts de la classe d'âge des hommes blancs de 18 à 35 ans. De nombreuses femmes de planteurs se retrouvent seules ou se 
regroupent entre elles sur des plantations qu'elles doivent diriger : c'est le «second front » de la guerre. D'autres femmes blanches sont contraintes à travailler, dans l'enseignement, l'infirmerie militaire ou les administrations de la Confédération, pour gagner leur vie. Ainsi, pour A. F. Scott - qui conduit jusqu'à son terme la thèse du "grand tournant» (watershed) - la guerre a eu un impact non seulement à court terme sur l'occupation des femmes de l'élite, mais aussi à long terme sur la condition des femmes blanches du Sud, qui, à la fin du XIXe siècle, récupèrent les fruits de leurs efforts pendant la guerre, en gagnant une certaine émancipation.

Ces deux thèses dominantes dans les années 1970 - un soutien sans faille des femmes de l'élite à la Confédération et l'émancipation des femmes pendant la guerre - ont été largement nuancées et remises en cause par des travaux plus récents, menés sur des échelles locales et des terrains précis. Jonathan Wiener (1977) ne comptabilise pas plus de femmes responsables de plantations en Alabama après la guerre qu'avant. Jean Friedman (1985) constate que la position des femmes dans les églises évangéliques est sensiblement la même avant et après la guerre. Aujourd'hui, les deux enquêtes de référence sur la question des femmes de l'élite pendant la guerre de Sécession ${ }^{46}$ - même si elles s'opposent sur un certain nombre de points ${ }^{47}$ - s'accordent pour remarquer les changements à court terme qu'apporte la guerre en termes de conditions de vie pour les femmes de l'élite. Cependant, ces travaux mettent l'accent à long terme sur les continuités dans la condition des femmes blanches du Sud. Comment comprendre cette différence entre court terme et long terme?

L'élément central de réponse réside dans la question des identités de genre, et notamment la permanence de l'identité de Lady durant la guerre. À première vue, à travers les conditions matérielles imposées par la guerre, et l'effondrement du monde qui les entoure, les femmes de la Confédération doivent écarter une partie des attributs qui les définissent en tant que Lady : toutes les femmes de planteurs doivent restreindre leurs dépenses, réduire leurs espoirs d'ascension sociale et surtout modifier leur représentation d'elles-mêmes, leurs attentes. Cependant, comme le souligne Drew Faust, si elles doivent refonder leur identité, c'est toujours en ayant profondément conscience de ce qu'elles ont à perdre dans cette redéfinition.

Ainsi, loin d'embrasser leurs nouvelles activités avec enthousiasme - comme le laisserait penser la thèse de l'émancipation féminine - les femmes de l'élite montrent une certaine réticence à réaliser ce qu'attend d'elles la Confédération. Les campagnes d'encouragement du textile maison, par exemple, se sont souvent soldées par des échecs car elles sont perçues par les planteurs (hommes et femmes) comme une atteinte à la santé et au statut des Ladies, rendues plus proches de leurs esclaves dans ce type d'activités.

39 Le modèle de la Southern Lady demeure ainsi en arrière-plan, même dans les situations les plus critiques, où la transgression des identités de genre parait la plus forte. C'est ce que montre l'épisode de la résistance des femmes de la Nouvelle-Orléans ${ }^{48}$. La ville est occupée dès 1862 par les troupes unionistes. Celles-ci doivent faire face à l'hostilité des habitants et tout particulièrement des femmes de la haute société, qui traitent l'armée d'occupation avec mépris, insultant les soldats et jetant des pots de chambre sur eux. L'attitude de ces femmes de la Nouvelle-Orléans paraît très ambiguë. D'une part, on peut considérer leurs actes comme éminemment politiques, ce qui constitue une profonde remise en cause de la définition de leur sphère d'activité et une recomposition de leur identité de genre face à l'ennemi. Mais d'autre part, le fait que 
ces femmes poursuivent leur résistance alors même que la répression unioniste s'est accentuée montre qu'elles se perçoivent comme au-dessus (ou à côté) des lois régissant la sphère publique, et sont perçues comme telles également par l'armée d'occupation : elles ne cessent d'être des Ladies.

La guerre, si elle modifie à court terme les conditions de vie des femmes blanches, ne transforme pas en profondeur les relations entre les sexes, car les conventions de genre et notamment l'identification au modèle de la Lady, demeurent inchangées. On peut même aller plus loin dans l'analyse : au moment où les Confédérés ne sont plus sûrs de la fidélité de leurs esclaves, il est d'autant plus important de compter sur la loyauté des femmes blanches dont on encense the very soul of the war. Le maintien des normes de genre constitue un enjeu politique pour la conservation de l'ordre social et racial du Sud lorsque celui est affaibli $^{49}$. Ce type de raisonnement nous place au cœur du programme énoncé par Joan Scott pour la gender history: il permet de renouveler la question de l'articulation entre genre, classe et race en l'intégrant pleinement dans l'histoire politique ${ }^{50}$. En analysant des configurations race/classe/genre (des constructions consubstantielles de rapports de pouvoir), nous avons mesuré combien les rapports de genre ne pouvaient être saisis que dans leur contexte racial et social d'énonciation; mais il s'agit de mesurer pleinement à présent l'impact des rapports de genre sur l'ordre social et racial du Sud, en recomposition autour de la guerre de Sécession.

Rhétoriques de virilité et rapports de race

41 La figure de la maisonnée, composée de dépendants (femmes et esclaves) sous l'autorité d'un patriarche, est emblématique de l'ordre social du Vieux Sud. Celle-ci conduit à nier toute masculinité aux hommes esclaves qui ne sont pas à la tête d'une maisonnée. Dans cette perspective, il n'est pas étonnant qu'abolitionnistes et unionistes aient utilisé la métaphore de la virilité pour enrôler des Noirs dans l'armée, à partir de 1862 : la rhétorique esclavagiste y est renversée, puisque les esclaves sont présentés comme des "hommes" (et non comme des enfants ou des animaux) luttant contre les confédérés qualifiés par exemple de "ratons laveurs" (coons) ${ }^{51}$.

Cette figure de la masculinité des esclaves, développée par les unionistes pendant la guerre civile et lors de l'émancipation, a eu des conséquences importantes en termes d'organisation familiale des Noirs dans l'après-guerre. Un des premiers objectifs des esclaves émancipés a consisté à établir des maisonnées: les familles séparées tentent de se recomposer, les couples revendiquent un mariage reconnu légalement et pour beaucoup l'émancipation signifie la fin du travail des femmes, à l'extérieur de la sphère domestique $^{52}$. Le thème $d u$ retrait des femmes noires du marché du travail est récurrent dans les écrits des planteurs - qui ont des difficultés à trouver des servantes et condamnent la paresse des anciennes esclaves qui «jouent» à la Lady - mais aussi des nordistes, qui craignent que la "fainéantise des femmes noires» ne menace l'expérience du libre travail salarié dans le Sud ${ }^{53}$.

43 Les historiens quantitativistes confirment l'idée présente chez les contemporains (les Blancs) de la baisse du travail des Noirs, qu'ils chiffrent à près du tiers ou du quart des valeurs d'avant la guerre. Les interprétations, cependant, divergent. Pour certains historiens $\mathrm{s}^{54}$ comme pour les contemporains, ce phénomène constitue un alignement des conventions de genre des Noirs sur le modèle de la classe moyenne et de l'élite blanche. Pour Jacqueline Jones (1985), le phénomène est plus complexe qu'un simple mouvement d'imitation. La réorganisation du travail des femmes est le fait à la fois des 
hommes et des femmes noirs. On ne peut donc ni conclure à la mise en place d'une pure domination masculine parmi les Noirs, ni à la thèse du matriarcat oisif des femmes. A fortiori, avec l'extension du métayage des Noirs (sharecropping), il est inadéquat de parler de retrait des femmes du marché du travail, puisque, en fait, elles continuent à travailler, mais dans le cadre familial. Certes, la mise en place de maisonnées noires permet aux hommes d'asseoir leur masculinité, mais les contraintes économiques, sociales et raciales imposent des ajustements en termes de normes de genre chez les anciens esclaves. Par exemple, les Blancs ne reconnaissent pas les noms que les Noirs se donnent et utilisent les noms des anciens propriétaires pour les désigner ${ }^{55}$. Si les femmes noires émancipées incorporent certains des privilèges qui étaient réservés jusque-là aux Ladies (comme le droit de placer les intérêts de leur famille au premier plan), le travail des femmes les plus pauvres, le moindre confinement domestique, l'importance du voisinage dans la vie privée, constituent autant de normes de féminité distinctes de celles des femmes blanches.

Cheerful Wife et Self-Made Man : des stéréotypes de genre qui participent au rétablissement de l'ordre social

Le modèle de la Southern Lady se trouve, à l'issue de la guerre de Sécession, transformé sans être totalement remis en cause. Laura Edwards ${ }^{56}$ examine l'idéal de féminité de l'élite blanche à travers une nouvelle, publiée dans un journal local de Caroline du Nord, en 1875 : Annie, the Butterfly. Annie, avant la guerre, est la femme d'un planteur riche et puissant. Elle est un papillon, une créature délicate, la fine fleur de la féminité du Sud. Cependant, la guerre conduit à la ruine de la plantation, ce qui oblige son mari à déménager en ville et tenter une carrière de juriste. Celui-ci échoue, est humilié et meurt rapidement. Annie, au contraire incarne le modèle de l'adaptation à un nouveau style de vie. Avec des moyens financiers limités, elle se concentre sur les tâches ménagères et domestiques, l'entretien de sa maison et se met même à travailler pour gagner sa vie.

Edwards qualifie l'idéal de la femme blanche de l'après-guerre de femme enjouée (Cheerful Wife): travailleuse, courageuse, dévouée à son intérieur et sa famille. Ce modèle paraît en continuité avec celui de la Southern Lady du Vieux Sud. Il emprunte les mêmes traits de l'éternel féminin. Cependant, ces valeurs ont un nouveau sens et aussi une nouvelle urgence dans le contexte de l'émancipation des Noirs, de la défaite de la Confédération et des difficultés économiques du Sud, contexte qui ébranle les fondements de l'identité de Lady. La période de la Reconstruction constitue en effet, du point de vue des femmes de l'élite blanche, un moment d'instabilité raciale et socioéconomique. Le passing, c'est-à-dire la possibilité pour un Noir de se faire passer pour blanc, est une crainte réactivée par l'émancipation. Comment une Lady peut-elle maintenir son statut en l'absence d'esclaves à sa disposition et à une période où il est difficile d'embaucher et retenir des domestiques? Comment demeurer une Lady alors qu'un certain nombre de familles de planteurs ont été appauvries, voire ruinées, pendant la guerre? Le personnage de la Cheerful Wife permet de répondre à ces questions en englobant des caractéristiques de travail et de courage dans sa définition.

En creux, se dessine aussi, à l'inverse du personnage du mari d'Annie, un nouveau type de masculinité. Il est nécessaire pour une femme, d'être épaulée par un homme qui surmonte les bouleversements de l'émancipation et l'effondrement politique de la Confédération. Alors que certains retrouvent une sorte d'éloge de la virilité dans le culte de la Lost Cause, d'autres diffusent de nouveaux modèles, venus du Nord. Edwards 
repère ainsi dans les journaux locaux, l'essor des Self-Made Men, de nouveaux capitaines d'industrie qui construisent leur richesse à la force de leur travail, en promouvant le changement économique, du chemin de fer aux nouvelles méthodes d'agriculture, par opposition avec les plantations héritées du Vieux Sud.

Les catégories de Cheerful Wife et de Self-Made Man incorporent des dimensions d'effort, de courage, de travail qui permettent d'intégrer en leur sein les hommes et les femmes de l'élite, ruinés pendant la guerre. En toute logique, on pourrait penser que cet élargissement par rapport aux figures de la Lady et du planteur gentleman permet une ascension sociale d'hommes et de femmes d'origine plus modeste, reposant sur le mérite. Or, il n'en est rien.

Il faut tout d'abord largement relativiser la pauvreté de l'élite blanche après la guerre : elle n'est pas vraiment pauvre (en tout cas beaucoup moins que les esclaves émancipés et que la plupart des Blancs), mais ne jouit plus des conditions matérielles qui lui permettraient à coup sûr de ne pas être confondue avec les couches sociales inférieures ${ }^{57}$. Les figures sexuées de la Cheerful Wife et du Self-Made Man apparaissent ainsi comme partie prenante des stratégies de distinction mises en œuvre par l'élite blanche, diminuée politiquement et économiquement lors de la guerre. Au lieu de mettre en avant directement des barrières de classe et de race (comme dans le système esclavagiste du Vieux Sud), les identités de genre lors de la Reconstruction, d'une manière plus subtile, plus cachée et plus pernicieuse maquillent ces inégalités en des différences "naturelles" ancrées dans le biologique. Les femmes sont identifiées en fonction de leur appartenance ou non à la catégorie morale des Cheerful Wives (vertueuses, courageuses, dévouées, maternelles, bien élevées, au tempérament égal). Or, cette appartenance repose en fait sur des conditions matérielles. Le recentrage sur la sphère domestique qui caractérise ces femmes vertueuses, par exemple, n'est rendu possible que par la possession de demeures spacieuses avec une division nette entre espaces privés et publics. Les femmes pauvres ou noires ne pouvant vivre que dans des maisons à pièce unique, paraissent a contrario rechercher la promiscuité, incapables d'entretenir leur intérieur, dénuées de goût. Autre exemple, les femmes de l'élite regardent avec mépris les dépenses en vêtements des Noires émancipées (le port de voilettes notamment) et condamnent celles-ci comme insolentes, agressives ou vulgaires $^{58}$. Elles sont désignées par nature comme des séductrices qui vivent dans la luxure, incapables d'entretenir une maison, alcooliques, agressives, de mauvaises mères ... portrait qui ne saurait satisfaire aux critères de l'éternel féminin.

Alors que les figures de genre de la Cheerful Wife et du Self-Made Man semblent fonctionner au mérite et sont potentiellement créatrices de mobilité sociale, les dés sont pipés. Les identités de genre sont prises dans des configurations socio-économique et raciale et redessinent, redoublent les distinctions de race et de classe qui par ailleurs (étant donné le contexte politique et économique) auraient tendance à se brouiller. Les genres - qui sont historiquement et culturellement variables, mais semblent toujours fixes et indiscutables à cause de leur référence à la nature, au corps - apparaissent comme une forme de langage du pouvoir ${ }^{59}$. Loin de constituer des conventions de genre "naturelles" ou "apolitiques", les figures de la Cheerful Wife et du Self-Made Man ont des retombées socio-économiques et raciales importantes.

50 Au-delà de l'apport immédiat en termes de découverte d'un champ relativement récent, marginal et donc peu connu et étudié en France de l'histoire américaine, que 
retirer d'un tel parcours à travers les travaux de la southern women's history pour une pratique des sciences sociales en France?

51 Sur le plan théorique et conceptuel, ce texte n'apporte aucune solution définitive, mais se veut plutôt comme la mise à jour d'un "chantier » pour les sciences sociales. Nous espérons que ce parcours à travers une historiographie américaine permet de dégager quelques voies pour des recherches à venir sur l'articulation d'inégalités sociales qui intègrent une analyse en termes de genre. Les questions d'articulations de différents types d'inégalités ne se réduisent pas, bien sûr, aux problématiques Race/Classe/ Genre : elles peuvent être posées également avec les variables âge, diplômes, religion, origine nationale, etc. suivant le contexte. Dans tous les cas, c'est la perception que nous avons des rapports de pouvoir ou des rapports de domination qui est en question.

Nous devons retenir le constat de l'impasse des approches en termes de sommation des atouts et des handicaps qui repose sur une "fiction » de la possible " désarticulation " des différentes inégalités sociales qui existeraient " toutes choses égales par ailleurs ». Les raisonnements du type construction consubstantielle de différents rapports de pouvoir paraissent plus solides. S'il est relativement aisé de définir théoriquement de tels ensembles, en utilisant notamment les concepts de configuration (cher à Elias), ou de système intégré (avancé par Danièle Kergoat), la manipulation pratique de ce type de concepts paraît plus délicate. Plus de vingt ans après le texte pionnier de Danièle Kergoat, qui proposait alors une articulation théorique entre sexe et classe sociale ${ }^{60}$, l'essentiel du travail de description empirique de situations concrètes qu'elle appelait de ses vœux pour faire travailler les concepts, reste à faire.

53 Nous voudrions ajouter qu'à une période où les sciences sociales ont tendance à oublier les stratifications socio-économiques - l'histoire sociale devient de plus en plus une histoire culturelle, la sociologie se désintéresse des classes sociales qui ont longtemps été au cœur de la discipline - comprendre l'imbrication des rapports de genre et des inégalités de classe peut constituer un renouveau pour penser la stratification socioéconomique. Comment comprendre la situation des pauvres sur le marché du travail si ce n'est en pensant que ce sont avant tout des femmes? Ou la diffusion des idées féministes dans la société si ce n'est en regardant la manière dont elles se traduisent dans les différents groupes sociaux ? Loin des préjugés de ceux qui ont longtemps pensé que s'intéresser au genre constitue immédiatement un oubli des problématiques en termes de classe, nous pensons que de telles approches n'appauvrissent pas, mais au contraire nourrissent et renouvellent notre réflexion sur les inégalités socioéconomiques.

\section{BIBLIOGRAPHIE}

ALEXANDER Adele Logan, 1992, Ambiguous Lives : Free Women of Color in Rural Georgia (1789-1879),

Fayetteville, University of Arkansas Press.

BLASSINGAME John, 1972, The Slave Community : Plantation Life in the Antebellum South, New York, Oxford University Press. 
-, 1977, Slave Testimony: Two Centuries of Letters, Speeches, Interviews and Autobiographies, BatonRouge, Louisiana State University Press.

BOURDIEU Pierre, 1998, La Domination masculine, Paris, Seuil.

BUTLER Judith, 1990, Gender Trouble. Feminism and the Subversion of Identity, London, New York, Routledge.

BYNUM Victoria, 1992, Unruly Women : The Politics of Social and Sexual Control in the Old South, Chapel Hill, University of North Carolina Press.

CASTRO Ginette, 1994, « De l'histoire des femmes à l'histoire des genres », in Jean Heffer et François Weil, Chantiers d'histoire américaine, Paris, Belin, pp. 309-339.

CLINTON Catherine, 1982, The Plantation Mistress : Woman's World in the Old South, New York, Pantheon.

CLINTON Catherine et SILBER Nina (dir.), 1992, Divided Houses : Gender and the Civil War, New York, Oxford University Press.

COTT Nancy, 1977, The Bonds of Womanhood: Woman's Sphere in New England, 1780-1835, New Haven, Yale University Press.

DAVIS Angela, 1971, « Reflections on the Black Woman's Role in the Community of Slaves », Black Scholar, vol. 3, n 4, pp. 3-15.

DELPHY Christine, 1970, « L'ennemi principal », Partisans.

DERRIDA Jacques, 1967, De la grammatologie, Paris, éditions de Minuit.

EDWARDS Laura, 1997, Gendered Strife and Confusion: The Political Culture of Reconstruction, Urbana et Chicago, University of Illinois Press.

-, 2000, Scarlett doesn't live here anymore: Southern Women in the Civil War Era, Urbana et Chicago, University of Illinois Press.

ELKINS Stanley, 1959, Slavery : a Problem in American Life, Chicago.

FAUST Drew Gilpin, 1996, Mothers of Invention: Women of the Slaveholding South in the American Civil War, Chapel Hill, University of North Carolina Press.

FOUCAULT Michel, 1976, Histoire de la Sexualité 1 : La volonté de savoir, Paris, Gallimard.

-, 1994, texte 306 in Daniel Defert et François Ewald (dir), Dits et écrits, vol 4, Paris, Gallimard, pp. 236-237.

FOX-GENOVESE Elisabeth, 1988, Within the Plantation Household: Black and White Women in the Old South, Chapel Hill et Londres, University of North Carolina Press.

-, 1991, Feminism without Illusions : A Critique of Individualism, Chapel Hill et Londres, University of North Carolina Press.

FRIEDMAN Jean, 1985, The Enclosed Garden: Women and Community in the Evangelical South 1830-1900, Chapel Hill, University of North Carolina Press.

GENOVESE Eugene, 1976, Roll, Jordan, Roll : The World The Slaves Made, New York, Vintage Books. GUTMAN Herbert, 1976, The Black Family in Slavery and Freedom, 1750-1925, New York, Pantheon Books. 
HALL Jacquelyn Dowd, 1992, « Partial Truths : Writing Southern Women's History » in Virginia Bernhard, Betty Brandon, Elizabeth Fox-Genovese, Thala Predue (dir.), Southern Women : Histories and Identities, Columbia et Londres, University of Missouri Press.

HEWITT Nancy, 1985, «Beyond the search for sisterhood : American Women's History in the 80 's », Social History, vol 10, pp. 299-321.

JONES Jacqueline, 1985, Labor of Love, Labor of Sorrow : Black Women, Work and the Family from Slavery to the Present, New York, Basic Books.

KERGOAT Danièle, 1978, « Ouvriers = ouvrières ? Propositions pour une articulation théorique de deux variables : sexe et classe sociale ", Critiques de l'économie politique, nouvelle série, $\mathrm{n}^{\circ} 5, \mathrm{pp}$. 65-97.

-, 1982, Les Ouvrières, Paris, le Sycomore.

LITWACK Leon, 1979, Been in the Storm So Long: The Aftermath of Slavery, New York, Kopf.

LESLIE Kent Anderson, 1992, « An Elite Mulatto Lady in the Nineteenth-Century Georgia » in Virginia Bernhard, Betty Brandon, Elizabeth Fox-Genovese, Thala Predue (dir.), Southern Women : Histories and Identities, Columbia et Londres, University of Missouri Press.

MASSEY Mary Elizabeth, 1966, Bonnet Brigades, New York, Knopf.

MCCURRY Stephanie, 1995, Masters of Small Worlds : Gender, Class and the Yeomanry of the South Carolina Low Country 1820-1860, New York, Oxford University Press.

MORTON Patricia (dir.), 1996, Discovering the Women in Slavery: Emancipating Perspectives on the American Past, Athens et Londres, University of Georgia Press.

NDIAYE Pap, 1994, «L'histoire afro-américaine » in Jean Heffer et François Weil, Chantiers d'histoire américaine, Paris, Belin, pp. 273-307.

PASSERON Jean-Claude et SINGLY François de, 1984, « Différences dans la différence : socialisation de classe et socialisation sexuelle », Revue française de Sciences Politiques, $\mathrm{n}^{\circ} 1, \mathrm{pp}$. 48-78.

RABLE George, 1989, Civil Wars: Women and the Crisis of Southern Nationalism, Urbana, University of Illinois Press.

SCOTT Anne Firor, 1970, The Southern Lady From Pedestal to Politics, 1830-1930, Chicago, University of Chicago Press.

- (dir.), 1993, Unheard Voices: The First Historians of Southern Women, Charlottesville, University Press of Virginia.

SCOTT, Joan Wallace, 1988a, Gender and the Politics of History, New York, Columbia University Press.

-, 1988b, « Genre. Catégorie d'analyse historique », Les cahiers du GRIF, n 37-38, pp. 1-20.

SINGLY François de, 1987, «Théorie critique de l'homogamie ", L'année sociologique, n 37, pp. 181-205

SMITH-ROSENBERG Carroll, 1975, «Female World of Love and Ritual : Relations between Women in Nineteenth-Century America », Signs 1, n 1, pp. 1-29.

STEVENSON Brenda, 1996, Life in Black and White : Family and Community in the Slave South, New York, Oxford University Press.

THEBAUD Françoise, 1998, Ecrire l'histoire des femmes, Paris, ENS éditions Fontenay-Saint-Cloud. 
WEBER Florence, 2002, « Pour penser la parenté contemporaine. Maisonnée et parentèle, des outils de l'anthropologie ", in Danièle Debordeaux et Pierre Strobel (coord.), Les solidarités familiales en questions. Entraide et transmission, Paris, LGDJ/MSH, coll. Droit et Société, Série Sociologie, vol. 34, $267 \mathrm{p}$.

WEINER Marli, 1997, Mistresses and Slaves : Plantation Women in South Carolina, 1830-1880, Urbana, University of Illinois Press.

WHITE Deborah Gray, 1985 (nouvelle édition : 1999), Ar'n't I a Woman : Female Slaves in the Plantation South, New York Norton.

WHITES LeeAnn, 1995, The Civil War as a Crisis in Gender : Augusta Georgia 1860-1890, Athens, University of Georgia Press.

WILEY Bell Irvin, 1975, Confederate Women, Westport, Conn., Greenwood.

WIENER Jonathan, 1977, « Female Planters and Planters' Wives in the Civil War and Reconstruction : Alabama, 1850-1870 », Alabama Review, XXX, pp. 135-149.

\section{NOTES}

1.Je ne m'attarderai pas, ici, sur le débat sexe / genre. J'emploierai par la suite indifféremment les deux termes. Si l'on considère le travail de dénaturalisation de la variable sexe comme totalement effectué, le sexe comme le genre sont traversés de part en part par le social (Butler 1990); d'où l'équivalence entre les deux termes.

J'emploierai dans la suite du texte le mot race sans précaution particulière, dans le sens américain de catégorie d'État. Il faudrait évidemment réfléchir dans un cadre français aux usages politiques de ce terme. La rédaction de cet article, lors de plusieurs étapes successives, a bénéficié des lectures attentives de Nancy Green, Eric Fassin et Baptiste Coulmont. Je les remercie pour leurs remarques constructives.

2.Singly $1987: 182$.

3.Delphy 1970.

4.À l'exception notable des travaux pionniers de Danièle Kergoat sur les ouvrières $(1978,1982)$ qui sont aujourd'hui "redécouverts" et sur lesquels je reviendrai.

5.La question de l'articulation est d'ailleurs reléguée en note de bas de page dans La domination masculine (Bourdieu 1998 : 26, note de bas de page $n^{\circ} 3$ ) : «Bien que, pour les besoins de la démonstration, j'aie été conduit à parler des femmes ou des hommes sans faire référence à leur position sociale, j'ai conscience qu'il faudrait prendre en compte, en chaque cas, et comme je le ferai plusieurs fois par la suite de ce texte, les spécifications que le principe de différenciation social fait subir au principe de différenciation sexuel (ou l'inverse) ».

6.Ce "saucissonage" institutionnel est cependant nuancé par le caractère souvent interdisciplinaire des programmes de «Cultural Studies ».

7.Scott 1988.

8.Ce n'est pas le lieu ici de présenter les apports du tournant linguistique en sciences sociales et toutes les critiques dont ce courant a été l'objet. Cf. Thébaud 1998 pour une remise en perspective éclairante de ce type d'approche.

9.Derrida 1967.

10.Foucault 1976, 1994. 
11.L'intitulé « autour de la guerre de Sécession » recouvre trois périodes historiques conventionnelles : «l'Antebellum Period » ou « Old South » jusqu'en 1860, la guerre de Sécession (1860-65) et la Reconstruction (1865-1877). La plupart des historiens, qui sont surtout des historiennes, ont travaillé à un moment ou à un autre sur ces différentes périodes, ce qui induit une continuité des références et des débats. Toutes les controverses ne seront pas traitées avec le même niveau de détail puisque je me suis attachée à cerner plus particulièrement la question de l'articulation entre race, classe et genre. Pour une bibliographie récente et exhaustive (mais non-thématique, ni commentée), on pourra consulter l'ouvrage de synthèse, Edwards 2000. On trouvera aussi deux bibliographies commentées dans Hall 1992 et Morton 1996, et en français, une remise en perspective de ces courants de recherche dans Castro 1994.

12.Des recherches isolées sur les femmes du Sud ont été menées dans les années 30 et 40, par des historiennes totalement marginalisées dans les universités du Sud-Est des États-Unis : Virginia Gearhart Gray, Marjorie Mendenhall, Julia Cherry Spruill entre autres. Comme le souligne Anne Firor Scott - qui a réalisé un travail de réédition de certains textes de ces pionnières (1993) : « No one was yet listening ».

13.La première Southern Conference on Women's History - conférence triennale organisée par la Southern Association for Women Historians a eu lieu en 1988.

14.Elkins 1959.

15.Le rapport publié en 1965 par le démocrate Daniel Patrick Moynihan, alors en poste au ministère du Travail, met l'accent sur les dysfonctionnements des familles noires américaines, et tout particulièrement la faiblesse des pères, à l'origine de la pauvreté des familles noires. Ce rapport a fait l'objet de nombreuses critiques de la part des militant(e)s des « Civil Rights ».

16.John Blassingame 1972, Eugene Genovese 1976, Herbert Gutman 1976 entre autres. Pour une approche synthétique en français, de ce courant, Ndiaye 1994.

17.De 1935 à 1943, dans le cadre du New Deal, le Works Progress Administration (WPA) établit des projets dans des domaines non-concurrentiels avec le secteur privé, afin de développer l'emploi public et réduire le chômage. Parmi ces projets, le Federal Writers Project (FWP) accorde des fonds à des écrivains et artistes. Une des grandes entreprises de FWP fut le financement d'une campagne d'interviews auprès de plus de 2000 anciens esclaves. De nombreux débats ont eu lieu dans la communauté historienne quant à l'usage que l'on peut faire de ces documents d'histoire orale, enregistrés plus de soixante ans après l'émancipation. Il faut prendre en compte notamment dans l'analyse de ces matériaux le caractère rétrospectif des récits sur l'esclavage, la position des enquêteurs (race, sexe, position politique) et replacer les entretiens dans le contexte politique, économique (la dépression), social, racial (la ségrégation) des années 30. Cf. John Blassingame 1977, pour un bilan des usages que l'on peut faire de ce type de matériaux.

18. White 1985.

19.Smith-Rosenberg 1975, Cott 1977. Pour une analyse critique de ce courant Hewitt 1985.

20.Hall 1992.

21.Scott 1970, Clinton 1982, Fox-Genovese 1988, Weiner 1997 principalement.

22.Davis 1971, Jones 1985, White 1985 entre autres.

23.À cet égard, la référence au best-seller de Margaret Mitchell, Gone with the Wind, paru en 1936, est centrale. De nombreux historiens font référence explicitement aux personnages du roman et/ou du film, pour construire, par contraste, leur vision du 
Vieux Sud. Un bon indicateur de la permanence de cette référence est le titre des ouvrages qui reprennent d'une manière ou d'une autre un élément du best-seller (Cf. Catherine Clinton, Tara Revisited, Abbeville, New York, 1995 ; Laura Edwards, Scarlett doesn't live here anymore, 2000).

24.À sa décharge, le singulier - "la femme" - était assez régulièrement utilisé en histoire des femmes dans les années 1970. Cf. Thébaud 1998.

25.Cet article a un statut particulier puisqu'il a été écrit par une philosophe, et militante communiste noire des Civil Rights. Angela Davis a participé au mouvement du Student Non-Violent Coordinating Commitee et des Black Panthers. Au moment où elle écrit cet article, elle est en prison, accusée d'avoir participé à l'évasion de prisonniers noirs. Ce n'est donc pas à proprement parler un travail d'historienne - elle n'a pas accès aux archives - mais plus de quinze ans avant le développement d'une histoire des femmes esclaves, A. Davis pose les termes du débat.

26.Bynum 1992, Mc Curry 1995.

27.Edwards 2000.

28.Stevenson 1996.

29. Alexander 1992.

30.Leslie 1992 relate l'histoire de la fille d'une esclave et d'un planteur, élevée par son père, en cachette, comme une Lady.

31.The Plantation Mistress est le deuxième ouvrage fondateur en Southern Women's History après celui de Scott. Alors que celle-ci se concentrait sur la période 1830-1930, Catherine Clinton travaille sur la période antérieure 1785-1835 (mais les conclusions qu'elle dresse semblent valoir pour toute la période antebellum, ce qui lui sera reproché). Pour la première fois, une historienne a dépouillé plus de 500 collections de manuscrits (journaux intimes et lettres) dans sept Etats du Sud. Cet ouvrage sera abondamment critiqué, pour la rapidité de ses analyses ( « les femmes blanches esclaves des esclaves », une thèse unilatérale et simpliste), qui s'appuient sur des sources à la fois trop larges (grand nombre de cas traités, mais assez rapidement) et trop restreintes (une étude des femmes les plus riches, dans la première période du Vieux Sud seulement). Surtout, la boîte noire de la domination masculine reste entière : l'oppression des femmes est pour C. Clinton un présupposé plutôt qu'un objet à analyser.

32.Aujourd'hui, il est attesté que les rapports sexuels entre femmes esclaves et planteurs constituaient la plupart du temps des viols. La question des sources est cruciale puisque l'on dispose de très peu de témoignages ou de traces écrites sur le sujet. La redécouverte de l'autobiographie de Harriet Jacobs en 1981 (la preuve de son authenticité longtemps déniée), une ancienne esclave qui subissait les viols de son maître et qui est parvenue à s'évader dans le Nord, constitue un tournant. On trouvera une analyse du cas Harriet Jacobs, dans l'épilogue de Fox-Genovese 1988.

33.Les sous-titres sont à cet égard explicite. The Southern Lady, from Pedestal to Politics d'A. F. Scott répond à l'ouvrage d'Aileen Kraditor, Up from the Pedestal : Selected Writing in the History of American Feminism, Chicago, 1968 qui développe la problématique des sphères séparées. Le sous-titre de la thèse de C. Clinton, Woman's World in the Old South, fait lui aussi référence à la métaphore spatiale d'un monde féminin à part.

34.Fox-Genovese 1991.

35.La notion de household est complexe à saisir et donc à traduire. C'est à la fois un concept (comme dans le livre de Fox-Genovese), mais aussi une catégorie « indigène » (catégorie de recensement, et de pensée pour les planteurs). J'ai choisi de le traduire 
par le terme maisonnée tel qu'il a été défini par F. Weber en 2002 : « groupe de résidence, doté d'une existence collective, analysable en termes de solidarité objective ".

36.Fox-Genovese sera cependant critiquée pour l'asymétrie de traitement des femmes blanches et des femmes noires. Si le plan de l'ouvrage se présente comme une alternance stricte de chapitres consacrés aux esclaves et aux planteurs, le livre s'arrête en général aux frontières de la Big House sans pénétrer dans le quartier des esclaves. Toutes ces critiques reflètent en fait un problème de sources : les journaux intimes et correspondances féminines sont nombreuses chez les femmes de planteurs, alors que les sources du FWP sont plus lacunaires et difficiles à interpréter. Ces critiques montrent aussi les limites de travaux au cadre d'analyse vaste : plusieurs plantations, sur différents Etats du Sud, dans un cadre temporel large, se fondant sur des sources traditionnelles. Les travaux plus récents adoptent des échelles locales afin d'étudier finement l'articulation des catégories de genre, de classe et de race. Cf. Weiner 1997, Edwards 1997, Stevenson 1996 par exemple.

37.Morton 1996, Weiner, 1997.

38.Davis 1971, Jones 1985.

39.White 1999: 5 « Qu'est-ce que cela signifiait pour les femmes noires de partager une certaine identité, mais aussi des handicaps à la fois avec les hommes noirs et avec les femmes blanches et en même temps d'être tout à fait différentes de ces deux populations?».

40.Fox-Genovese 1991.

41. White 1999: 5 .

42. White 1999 : 4 .

43.Danièle Kergoat travaille sur l'articulation théorique des variables sexe et classe sociale pour décrire les ouvrières des années 1970. «C'est en cela qu'il y a autonomie de la situation des femmes et des hommes de la classe ouvrière : dans le cas de la force de travail féminine, les mécanismes d'exploitation copient, utilisent et redoublent les mécanismes de domination; du même coup exploitation et domination forment un système intégré où la marge de liberté des femmes (en tant que femme, en tant que travailleuse) est extrêmement réduite [...]. Dès lors, le concept de surexploitation devient insuffisant pour rendre compte de la réalité, puisque les phénomènes de discrimination ne sont pas seulement quantitatifs mais aussi qualitatifs (les mouvements de déqualification et de surqualification ne sont pas plus ou moins forts selon les sexes, ils vont dans des sens différents) » (1978: 76-7).

44.Jusqu'à la fin des années 80 , il n'allait pas du tout de soi que l'histoire sociale (critique de toute histoire événementielle), et en particulier l'histoire des femmes s'empare de l'étude de la guerre civile, chasse gardée de l'histoire militaire et politique. Comme le regrette George Rable, en 1989, les deux histoires s'écrivent encore de façon cloisonnée, dans un mépris partagé.

45.Massey 1966 ; Scott 1970 ; Wiley, 1975. On peut voir dans ces ouvrages, qui encensent le rôle des femmes dans la guerre de Sécession, les derniers prolongements des écrits de la Lost Cause. (Whites 1995).

46.Rable 1989 ; Faust 1996.

47.En particulier, alors que George Rable affirme que les femmes de l'élite restent fidèles tout au long de la guerre à la cause confédérée, Drew Faust montre combien elles perdent progressivement la foi dans la Confédération et s'opposent même à la guerre à mesure que les conditions matérielles deviennent plus difficiles. 
48. Rable 1989.

49. Whites 1995.

50.Scott 1988.

51.Clinton et Silber 1992.

52.Gutman 1976.

53.Jones 1985.

54.Litwack 1979.

55.Edwards 1997.

56.Edwards 1997. Cet ouvrage est représentatif des recherches les plus récentes qui procèdent à l'analyse fine de configurations dynamique race/classe/genre sur une échelle locale : le comté de Granville en Caroline du Nord, pendant la période de la Reconstruction. Les innovations sont d'ordre méthodologique avec l'examen attentif et le traitement original des archives : textes de loi, sources judiciaires, presse locale ou encore recensement. Ce travail présente aussi un intérêt sur le plan des problématiques puisqu'il s'agit de repenser à partir du triptyque race/classe/genre l'articulation entre l'histoire sociale et l'histoire politique de façon inédite, en remettant en cause des oppositions classiques comme public/privé.

57.Edwards 1997.

58.Jones, 1985

59.Scott 1988

60.Kergoat 1978.

\section{RÉSUMÉS}

Race/classe/genre. Parcours dans l'historiographie américaine des femmes du Sud, autour de la guerre de Sécession : comment articuler des inégalités de genre avec d'autres types d'inégalités sociales? Au lieu de proposer une (improbable) théorie de l'articulation, j'interroge ici un champ historiographique particulier où a été mis en œuvre pratiquement ce type de questionnements. Ce texte peut être lu comme un review article qui fait le point sur les débats intellectuels dans un champ de l'histoire des femmes, peu connu en France : l'histoire des femmes du Sud est un domaine récent de l'histoire américaine, confronté dès sa naissance à l'articulation de rapports de pouvoirs fondés sur la race, le genre et la classe et qui présente une très forte " cumulativité " dans ce domaine. Ce parcours dans une historiographie américaine ne propose pas de solution théorique définitive aux questions d'articulation de différents types d'inégalités, mais il souhaite fournir, quelques jalons et pistes de travail utiles pour les sociologues et historiens, qui se posent ces questions.

Race/Class/Gender. Exploring Southern American women's history : how do gender and other social inequalities interact with one another? In order to articulate to articulate Race, Class and Gender I'd rather not come up with an (unlikely) theory of interaction. Rather I shall focus here on a specific moment in the South, from the Antebellum years to Reconstruction. This article can first be read as a review of southern women's history, as its recent historiography is still not well known in France. Southern women's history is a quite recent field of research in American history and it has always been confronted to the interaction of power relations based on race, 
gender and class. Beyond providing historical landmarks necessary to understanding the period, this article deals with larger theoretical issues. Although in the final analysis I shall not suggest a theory that would articulate gender with other social inequalities, I hope that the spreading of these issues will provide sociologists and historians with useful tools and landmarks.

\section{AUTEUR}

\section{CÉLINE BESSIÈRE}

Céline BESSIERE, allocataire monitrice à l'Université de Marne-la-Vallée et doctorante au Laboratoire de Sciences Sociales (Jourdan), fait une thèse de sociologie sur les exploitations familiales viticoles de la région de Cognac qui intègre une analyse en termes de genre. 